\title{
Former des acteurs de changement à l'université dans le contexte d'une transition écosociale
}

Mélanie Champoux

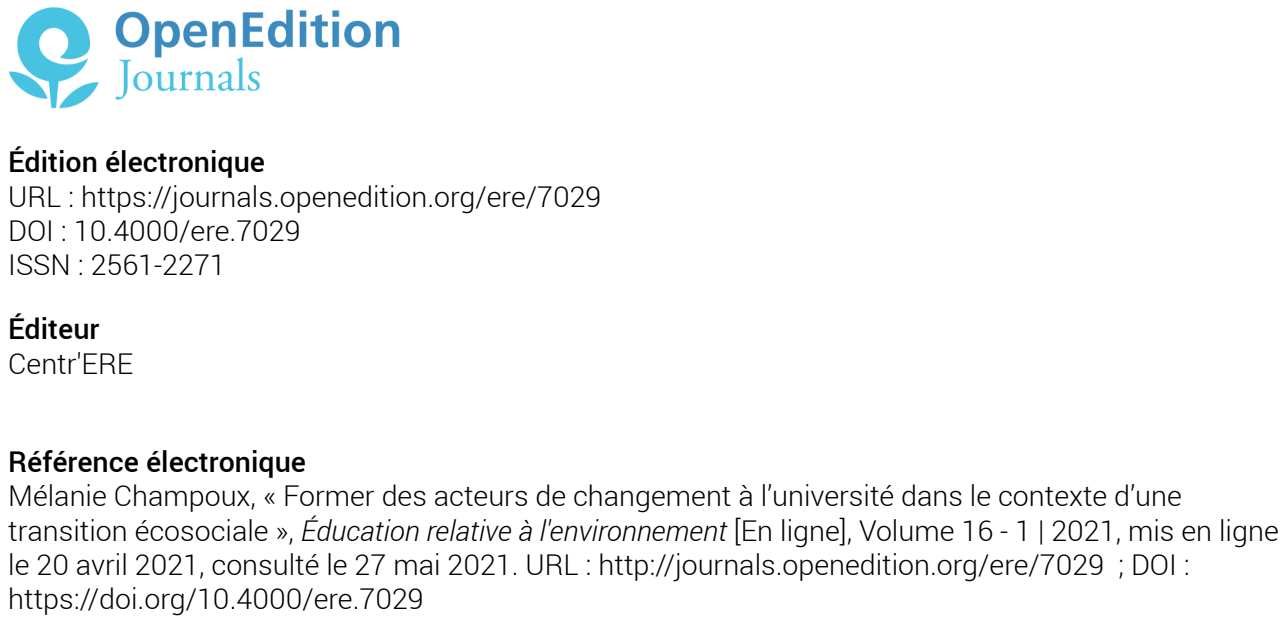

Ce document a été généré automatiquement le 27 mai 2021. 


\title{
Former des acteurs de changement à l'université dans le contexte d'une transition écosociale
}

\author{
Mélanie Champoux
}

1 Dans le contexte d'une crise socioécologique majeure comme celle à laquelle sont désormais confrontées nos sociétés, former des acteurs de changement à l'université apparait, pour le moins, une orientation éducationnelle accrocheuse. Mais pour que cela ne demeure pas qu'un simple slogan visant à positionner les universités sur la scène compétitive de l'éducation supérieure, il convient de nous y attarder avec criticité. Nous pouvons notamment nous demander: que signifie être un acteur de changement? Pour qui et pour quoi former des acteurs de changement? Au nom de quelles valeurs et de quels principes? Comment y arriver?

2 Cet article a pour objectif de communiquer de manière concise les résultats d'une recherche réalisée dans le cadre d'un essai de maitrise en environnement à l'Université de Sherbrooke (Champoux, 2021). Par-delà, il vise à proposer des repères théoriques et pratiques pour inspirer et appuyer le développement et la mise en œuvre de programmes universitaires orientés envers la formation d'acteurs de changement, et ce, dans une diversité de programmes et de disciplines.

3 Tout d'abord, sont exposés les principaux éléments de la problématique en question afin 1) de mieux comprendre le contexte de crise socioécologique dans lequel s'inscrit la mouvance observée autour du terme "formation d'acteurs de changement» et 2) d'identifier les principales lacunes et limites théoriques et pratiques que nous observons relativement à l'élaboration et la mise en œuvre de certaines formations dites d'acteurs de changement en contexte non formel et en milieu formel. Les objectifs de notre recherche sont ensuite précisés. Puis, après avoir explicité les principales étapes méthodologiques mises en œuvre, nous présentons un condensé des résultats issus des volets théorique et empirique de la recherche. Finalement, nous exposons les principaux traits de notre proposition de repères théoriques et pratiques pour penser 
et mettre en œuvre des formations d'acteurs de changement en milieu universitaire dans le contexte d'une transition écosociétale.

\section{Problématique}

4 Afin de faire état de la problématique, nous présentons d'abord le contexte général de crise socioécologique dans lequel elle s'inscrit. Puis, nous explicitons les limites et les lacunes que nous avons constatées à travers notre analyse de différentes formations d'acteurs de changement en milieu formel et non formel.

\section{Une crise plurielle dans une société postmoderne}

5 Nos sociétés sont aujourd'hui interpelées de toutes parts et de manière aigüe par un ensemble de crises interreliées : crise écologique, crise climatique, crise énergétique, crise économique, crise sociale, crise sanitaire, crise de la démocratie, crise de sens, crise de l'éducation, etc. Non seulement une telle crise socioécologique globale est empreinte d'incertitude, mais elle se caractérise par ses dimensions de gravité et d'urgence.

6 Dans ce contexte d'une telle ampleur et d'une extrême complexité, il devient difficile pour chaque individu d'identifier les causes profondes de ces maux et d'agir en conséquence pour leur résolution. D'autant plus qu'en raison de l'effritement de nos relations aux institutions et de l'effondrement de nos certitudes sur le monde (Labadie, 2015), il devient incertain de nous prononcer sur la nature exacte des changements à opérer et sur les manières d'y arriver. Par ailleurs, si nous comprenons que nous devons mettre en œuvre des solutions collectives, nous sommes toutefois aux prises avec une multiplicité de points de vue d'une part, et avec l'individualisme d'autre part.

7 C'est dans ce climat, que d'aucuns qualifieraient d'anxiogène, que les étudiants devraient désormais accomplir leurs études universitaires et apprendre à penser et à agir comme acteurs de changement.

\section{Former des acteurs de changement : une notion polysémique séduisante}

8 Il semble en effet que c'est dans ce contexte que nous voyons aujourd'hui apparaitre un nombre de plus en plus grand de programmes universitaires et de formations en milieu non formel qui promeuvent une formation d'acteurs de changement (Delafield et Mukherjee, 2016; The changemaker project, 2017; Changemaker residency, 2019; Bonnici, 2020 ; Echoingreen, 2020 ; Hackwood college, 2020). Face aux problématiques socioécologiques, certaines organisations éducatives telles qu'Ashoka invitent le monde de l'éducation à «équiper les jeunes avec les outils et l'état d'esprit nécessaires pour créer un monde meilleur» (AshokaU, s.d; Fuessel, 2020). Si un "monde meilleur» constitue une expression polysémique et " acteur de changement ", un mot-valise, une recherche sur Internet a permis de constater que les programmes de formation qui emploient le terme " acteur de changement» (ou «changemaker »), mettent de l'avant de nombreux autres termes polysémiques à forte charge positive tels qu'innovation, leadership, empowerment, coconstruction, etc. Ces termes n'étant pas définis clairement et les fondements axiologiques qui les sous-tendent n'étant pas explicités, un flou 
demeure quant à leurs intentions profondes et à la vision du monde qu'ils défendent. De quels acteurs de changement et de quel monde meilleur est-il question dans ces programmes de formation?

\section{Des formations en manque de repères}

9 Afin de mieux comprendre les fondements implicites aux formations d'acteurs de changement telles que pratiquées actuellement (principalement dans le monde anglosaxon), nous avons effectué une recherche documentaire et nous avons analysé le discours employé par ces programmes. Notre analyse montre que la très grande majorité de ceux-ci emploient des termes issus du monde de l'entrepreneuriat social (design thinking, prototypage, pitch, etc.). L'influence de l'organisation Ashoka fondée par l'entrepreneur social Bill Drayton y est manifeste. Or, il nous apparait que si l'entrepreneuriat social peut apporter une contribution à la résolution des problématiques socioécologiques, il ne peut toutefois pas en constituer l'unique solution. Former des acteurs de changement ne saurait se réduire à former des entrepreneurs sociaux.

Nous constatons donc que, malgré la popularité grandissante de ce type de formations en milieu non formel et son intégration de plus en plus grande dans le milieu de l'éducation formelle, ce foisonnement d'initiatives n'est pas accompagné par le développement de repères théoriques et pratiques sur lesquels appuyer leur développement et leur mise en œuvre. Ce qui se donne à voir ressemble davantage à un ensemble de pratiques à la pièce, suivant l'air du temps.

\section{Question de recherche et objectif principal}

11 Constatant que les formations d'acteurs de changement offertes en milieu formel et non formel échouent à fonder leurs pratiques sur des propositions et des assises théoriques inspirantes, cohérentes entre elles et pertinentes au regard des enjeux posés par le contexte de crise socioécologique et la transition à réaliser, nous posons la question de recherche suivante :

Quels devraient être les fondements théoriques et les options pratiques d'une formation d'acteurs de changement en milieu universitaire dans le contexte d'une transition écosociétale ${ }^{1}$ ?

Notre principal objectif est donc de définir et de justifier des bases théoriques et pratiques visant la modélisation d'une proposition de formation d'acteurs de changement en milieu universitaire dans le contexte d'une transition écosociétale. Cette modélisation permettra de clarifier les dimensions formelle, axiologique, praxéologique et explicative (soit les dimensions constitutives d'une théorie en éducation) (Maccia, 1966; Legendre, 2005) et ouvrira ainsi sur une diversité de possibilités créatives et contextuellement adaptées en matière de pratiques éducatives universitaires. 


\section{Méthodologie}

14 Afin d'expliciter notre méthodologie de recherche, nous apportons d'abord quelques éléments clés qui précisent le type de recherche effectuée, puis nous présentons succinctement le processus de collecte et d'analyse de données mis en œuvre dans le cadre de la réalisation du volet théorique puis du volet empirique de notre démarche de recherche.

\section{Type de recherche}

Cette recherche qualitative de type interprétative critique qui s'inscrit dans le courant de la critique sociale (Habermas, 1971) adopte une épistémologie de type intersubjectiviste et dialectique. La méthodologie employée s'inspire du processus général d'anasynthèse (Messier et Dumais, 2016) qui consiste à effectuer de fréquents allers-retours entre analyse et synthèse de nouvelles données entrantes. Il s'agit dans notre cas d'un processus qui combine un volet théorique et un volet empirique.

\section{Collecte et analyse de contenu}

16 Le processus méthodologique mis en œuvre dans le cadre de cette recherche présente l'originalité de l'interrelation entre un volet théorique et un volet empirique. Le dialogue entre ces deux volets permet d'ancrer notre réflexion dans une compréhension partagée par d'une part, des philosophes, sociologues et pédagogues critiques et d'autre part, des acteurs de terrain concernés en priorité par les propositions à formuler.

\section{Volet théorique}

17 L'exploration du champ notionnel relatif aux termes "acteurs de changement » et «transition écosociétale» ainsi que la lecture flottante de documents s'y rattachant font apparaitre la prédominance de quatre champs théoriques contributifs à notre objet de recherche. Il s'agit des champs de l'éducation relative à l'environnement (ERE), de l'éducation conscientisante et émancipatrice, de l'éducation à la démocratie et à la citoyenneté et du champ de l'innovation sociale. En accord avec nos présupposés paradigmatiques qui ancrent notre réflexion dans une perspective sociocritique et qui, dans la lignée des pédagogues critiques, envisagent le rôle de l'éducation en tant que force de transformation des réalités sociétales, nous avons consulté et mis à contribution certains des auteurs critiques majeurs de ces quatre champs. Le corpus de documents sélectionnés a fait l'objet d'une analyse qualitative de contenu (Mucchielli, 2009, p. 371-372). Les contributions ont été analysées et catégorisées selon les dimensions constitutives d'une théorie en éducation telles qu'identifiées par Renald Legendre $(1985,2005)$ : dimensions formelle, axiologique, praxéologique et explicative ${ }^{2}$. Celles-ci ont ensuite été interprétées selon un processus inductif et synthétisées de manière dialectique, permettant ainsi de faire ressortir les éléments convergents et complémentaires entre eux. 


\section{Volet empirique} programmes et de disciplines à l'Université de Sherbrooke. Les entretiens ont permis de recueillir leurs perceptions et leurs aspirations relativement à une formation d'acteurs de changement. Les verbatims d'entretien ont été soumis à une analyse qualitative de contenu afin de mettre en exergue les dimensions constitutives d'une formation d'acteurs de changement en milieu universitaire dans le contexte d'une transition écosociétale.

lement, une synthèse interprétative, critique et dialectique a été effectuée entre le volet théorique et le volet empirique permettant ainsi de formuler une proposition modélisée de repères théoriques et pratiques.

\section{Résultats des volets théorique et empirique}

Dans cette section, nous présentons un condensé des principales données que nous avons d'abord recueillies, puis analysées et enfin synthétisées dans le cadre de la réalisation $\mathrm{du}$ volet théorique, puis $\mathrm{du}$ volet empirique de notre démarche de recherche.

\section{Volet théorique}

21 Cette section présente un condensé des synthèses issues du volet théorique selon qu'elles éclairent les dimensions formelle, axiologique ou praxéologique d'une formation d'acteurs de changement en milieu universitaire dans le contexte d'une transition écosociétale $e^{4}$. Pour chaque dimension, un paragraphe présente une synthèse de la contribution de chacun des champs contributifs. Les principaux auteurs ayant été convoqués dans les significations qui sont évoquées sont cités de manière agrégée à la fin de chacune des sous-sections. ${ }^{5}$

\section{Dimension formelle}

L'ERE concerne le développement intégral des personnes dans toutes leurs dimensions. Il ne s'agit pas d'une éducation thématique. L'ERE a pour objet l'harmonisation du réseau de relations entre les humains et entre les humains et l'environnement. L'ERE des adultes se rapporte à un processus actif de co-apprentissage tout au long de la vie, à propos, dans, par et à travers l'environnement. Les relations à l'environnement des humains sont améliorées à travers le développement d'une conscience et d'un agir critique, individuel et collectif. (Sauvé, 1997 ; Sauvé et Asselin, 2018 ; Villemagne, 2008, 2017)

L'éducation à la citoyenneté et à la démocratie concerne le développement d'une conscience critique à l'égard des injustices sociales et environnementales, et ce, par l'analyse des causes profondes et systémiques à l'origine des maux de société. L'insertion active des citoyens (individus et collectivités) dans la société pour la rendre plus juste se réalise à travers un processus émancipatoire. Cette éducation est concernée par la capacité des individus et des groupes sociaux à mettre à distance 
critique ses désirs (versus ses besoins) pour entrer en dialogue avec le monde. (Westheimer et Kahne, 2004 ; Carr, 2011 dans Thésée, Carr et Potwora, 2016)

À l'opposé d'une éducation bancaire, l'éducation conscientisante et émancipatrice s'inscrit dans un processus de co-construction des savoirs entre l'éducateur et les apprenants. Le monde (les réalités sociétales et environnementales) est au centre de la situation d'apprentissage. C'est dans, par et à travers ces réalités que les coapprenants se rencontrent et s'engagent pour s'expliquer le monde de manière critique et que leur parole dialogique constitue une praxis par laquelle action et réflexion ne sont plus qu'un seul et même processus dialectique qui contribue à l'émancipation et à la transformation individuelle et sociale. L'éducation transforme les personnes et transforme le monde. (Freire, 1974 ; Shor, 1992 ; Kincheloe, 2004)

L'innovation sociale s'attarde à la création et la mise en œuvre « durable » de nouveaux arrangements sociaux en réponse à des besoins non satisfaits ou à des injustices. Ces nouveaux arrangements sont portés par des acteurs qui se posent en rupture critique avec les structures sociétales dominantes. Les formes que peut prendre l'innovation sociale sont multiples (entreprise sociale, modèle de gouvernance, discours de résistance, etc.) et elles peuvent être réalisées à plusieurs échelles de la société. (Bélanger, 2007 ; Fontan, 2007 ; Nussbaumer et Moulaert, 2007)

\section{Dimension axiologique}

L'ERE assume résolument son caractère politique. Les objectifs de l'ERE sont en lien avec le développement de connaissances (savoirs écosystémiques), d'attitudes (participation active, engagée et critique, congruence), de qualités et de valeurs (compassion, empathie, altruisme) et de compétences (habiletés de résolution de problèmes environnementaux, écogestion) favorables à l'optimalisation du réseau de relations personnes-société-environnement. Le développement de la conscience critique apparait comme un pilier fondamental pour articuler prise de conscience, engagement lucide, autonome et authentique envers la transformation des réalités non désirables. (Sauvé, 1997, 2011; Thésée et Carr, 2008; Clover, Jayme et Hall, 2013 ; Villemagne, 2017)

Pour l'éducation à la citoyenneté et à la démocratie, l'objectif principal qui est celui du "vivre ensemble" suppose la capacité de se détacher de Soi pour entrer dans un dialogue authentique et une praxis avec l'Autre. Ce qui est visé concerne à la fois l'autonomie, la responsabilité et la solidarité des sujets acteurs qui doivent prendre une part active dans l'avènement d'un monde plus juste et équitable pour tous. (Westheimer et Kahne, 2004 ; Biesta, 2006, 2017)

28 L'éducation conscientisante et émancipatrice vise le "plus-être » des personnes, leur humanisation. Ce "plus-être " se construit notamment à travers l'émancipation. La finalité relative à la libération de tous les peuples se déploie à travers une pédagogie critique qui cherche à développer la conscience critique des personnes et à stimuler leur engagement solidaire envers la justice sociale. Pour arriver à s'émanciper et à transformer les réalités injustes, les personnes doivent allier action et réflexion dans un seul et même mouvement (praxis). Par ailleurs, il importe de stimuler les habiletés créatrices afin que les personnes puissent dépasser leur attitude de fatalité et imaginer d'autres possibles. (Freire, 1974 ; Shor, 1992) 
29 L'innovation sociale doit permettre une participation élargie, plus équitable et démocratique des personnes à la résolution des problématiques qui les concernent. La finalité qui est celle de construire un monde caractérisé par une plus grande justice sociale et environnementale (par ailleurs indissociables) doit se déployer sous l'impulsion d'initiatives orientées notamment envers l'édification d'une économie plurielle, voire post-capitaliste. Pour y arriver, l'innovation sociale doit chercher à mettre en œuvre des mécanismes institutionnels permettant aux multiples initiatives de constituer une réelle alternative au modèle sociétal dominant. L'innovation sociale cherche à favoriser le développement de l'esprit critique (qui consiste notamment à comprendre la racine des problématiques, les causes profondes), le leadership, la créativité, l'engagement social et la solidarité. Au-delà des visées de transformation structurelles, l'innovation sociale est aussi tournée vers la création d'un monde plus beau, plus responsable, humain et convivial. (Thésée, Carr et Potwora, 2016; Chateauvert, Dufort, Durand Folco, Morales Hudon, Stambouli, Tremblay-Pépin et Wilson, 2020)

\section{Dimension praxéologique}

30 Traversée historiquement par une diversité de courants (naturaliste, conservationniste, systémique, scientifique, mésologique, moral/éthique, holistique, ethnographique, de l'écoformation, biorégionaliste, praxique, de la critique sociale, féministe, de la durabilité/soutenabilité et de l'écocitoyenneté), l'ERE mise sur une complémentarité d'approches pédagogiques: expérientielle, holistique, systémique, interdisciplinaire, coopérative, critique, résolutique, créative et artistique. La multiplicité des approches permet de développer plusieurs des dimensions de la personne : cognitive, affective, morale, sociale, spirituelle, etc. Une diversité de stratégies pédagogiques permet d'atteindre l'ensemble des objectifs. Les éducateurs et les apprenants coconstruisent activement et de manière critique les savoirs dans le creuset de l'environnement. L'apprentissage est actif et interactif et favorise l'expérimentation. La construction et l'expression des savoirs peuvent non seulement s'actualiser à travers la lecture et l'écriture, mais également à travers les arts. Les rapports entre les sujets et l'agent de la situation éducative sont basés sur la réciprocité et la mutualité. (Sauvé, 1997, 2017 ; Clover et coll., 2013)

31 La discussion en groupe constitue sans doute la méthode pédagogique privilégiée par l'éducation à la citoyenneté et à la démocratie. Elle doit permettre l'apprentissage du dialogue authentique, éthique, respectueux de l'autre et tendu vers la compréhension mutuelle. Elle doit être animée afin d'approfondir les réflexions de manière critique et favoriser la confrontation de points de vue différents. Les participants à la discussion de groupe doivent être encouragés à s'engager dans la discussion et ce, par une écoute et une parole active et responsable. D'autre part, l'apprentissage de la vie démocratique peut s'effectuer à travers la discussion grâce à la mise au jour des relations de pouvoir et des inégalités inhérentes au groupe de discussion lui-même et l'engagement conjoint de tous à faire échec à ces dynamiques injustes. L'éducateur n'est pas un simple facilitateur, il doit prendre une part active dans la discussion, mais il doit savoir doser ses interventions. (Kelly et Brandes, 2001; Lefrançois, Éthier et Demers, 2009 ; Martineau et Simard, 2010)

32 Pour l'éducation conscientisante et émancipatrice, le processus pédagogique critique démarre avec la recherche des thèmes générateurs des apprenants. À partir de leur 
univers thématique (ce qui les habite au quotidien, ce qui les interpelle, les inquiète, etc.), l'éducateur construit le programme éducatif. C'est par l'intermédiaire de l'expérimentation et de l'analyse des réalités (qui sont au centre de la situation éducative) que les savoirs se coconstruisent. L'ensemble du processus éducatif s'appuie sur la dialectique entre subjectivité et objectivité. Ainsi, la parole qui émerge est critique et dialogique. Cette parole est celle d'une personne transformée (qui se libère et s'émancipe) et elle constitue une parole engagée envers la transformation du monde. La réflexion s'effectue à travers l'action et l'action est guidée (voire impulsée) par la réflexion: c'est la praxis. La réflexion critique doit soulever les injustices sociales et environnementales et s'engager envers la dénonciation, la résistance, le requestionnement, la re-création et la ré-affirmation d'un monde plus juste et équitable pour les êtres humains et pour le reste de la Nature. La recherche d'alternatives doit être encouragée. L'éducateur est coapprenant et les apprenants sont coéducateurs. L'éducateur demeure toutefois dans une relation asymétrique avec les apprenants : son autorité est démocratique et bienveillante et ne doit pas être confondue avec un laisser-faire ni avec l'autoritarisme. (Freire, 1974; Shor, 1992; Thésée et Carr, 2008 ; Pérez-Ibanez, 2018)

Pour le champ de l'innovation sociale, l'éducateur doit incarner une attitude d'espoir en un monde meilleur et de confiance envers les apprenants qui sont vus comme des sujets historiques créateurs et re-créateurs des réalités sociétales. L'imagination et la créativité sont mobilisées conjointement avec les valeurs et l'analyse critique afin de proposer d'autres possibles. L'éducateur doit savoir équilibrer et partager cette vision optimiste avec une approche critique des réalités. Pour cela, il doit amener les apprenants à défier les réalités, à les questionner, à comprendre les obstacles et les leviers du changement. Les approches éducatives privilégiées sont les approches systémique, critique, expérientielle, coopérative et résolutique. L'approche transdisciplinaire - vue comme une enquête à propos d'un domaine d'objets (Dewey, 2014) dans laquelle la réalité est étudiée à travers le prisme de l'expérience vécue (Dewey, 2005) - est inhérente à l'ensemble de la démarche éducative. (Chateauvert et coll., 2020)

\section{Volet empirique}

34 Cette section présente un condensé des synthèses issues des contributions des participants : enseignants et étudiants. Certains extraits de verbatims sont rapportés afin de mieux illustrer les propositions des participants et d'appuyer l'interprétation que nous en faisons.

\section{Contribution des enseignants}

Cette section présente de manière synthétique les principaux apports des entretiens réalisés avec huit enseignantes et enseignants œuvrant chacun dans un domaine de formation différent: gestion, économie, droit, philosophie, sciences de la santé, environnement, éducation et travail social. De manière cohérente, ces apports sont présentés selon qu'ils éclairent la dimension formelle, axiologique ou praxéologique de l'éducation envisagée. 


\section{Dimension formelle}

Un acteur de changement est une personne qui a développé une conscience critique sociale, qui est animée par une volonté d'agir pour transformer les réalités sociétales (incluant la dimension environnementale qui lui est inhérente) non désirables. «C'est quelqu'un qui va s'impliquer, qui va être conscient de son rôle de professionnel. C'est quelqu'un qui s'implique pour changer la société, aider au changement des individus pour s'en aller vers quelque chose de mieux. » (Mireille)

C'est aussi quelqu'un qui cherche, à travers son émancipation et son alliance avec l'Autre, à tendre vers un plus-être.

L'acteur de changement c'est quelqu'un qui devient de plus en plus conscient de ce qu'il veut et de ce qu'il veut être et non pas d'être un objet formaté par d'autres pouvoirs [...] il recherche à être plus vivant [...] il cherche sa capacité à exister plus fortement. Alors l'acteur de changement veut essayer de développer qui il est, les potentialités qu'il a en lui [...]. (Jocelyn)

39 Le changement n'est pas linéaire et ne doit pas être dirigé par un petit groupe de leaders. Le changement doit être émergent et provenir de la base. «Le nouveau paradigme du changement, c'est l'émergence. Le changement c'est l'émergence de ce qui est là, ce qui est en relation génère du changement. » (Simon)

\section{Dimension axiologique}

Une formation d'acteur de changement doit viser le développement de la conscience critique sociale et des habiletés relatives à la construction d'un argumentaire solide et bien étayé. Luc exprime cette idée en disant que les personnes apprenantes doivent devenir « [...] capables de regarder une situation actuelle, de voir quelles sont ses failles - puis de s'en aller vers le changement.». Cet enseignant poursuit en détaillant davantage son orientation éducationnelle :

1 Pour moi, ce qui est important pour être un acteur de changement c'est d'aller au-delà des opinions - être capable de développer un argumentaire et de l'étayer. Et c'est à partir de là qu'on établit notre propre crédibilité et c'est avec cette crédibilité-là qu'on est capable de faire changer les choses. Parce que des opinions, il y en a plein les réseaux sociaux. (Luc)

De plus, les étudiants doivent apprendre à dialoguer avec l'Autre (autres disciplines et autres types de savoirs) pour arriver à co-construire leurs savoirs. Aussi, il est nécessaire de développer la capacité réflexive et métacognitive des étudiants et ce, de concert avec le développement de leur volonté d'agir, de s'engager en cohérence avec leurs valeurs profondes, avec ce qui a du sens pour eux. Ainsi, il devient également nécessaire de les amener à clarifier leur système de valeurs et à mieux prendre conscience de leur intentionnalité. De plus, l'accent doit être mis sur le développement des capacités créatrices. Les étudiants doivent être amenés à imaginer d'autres possibles. La compréhension des rapports de pouvoir et la confiance en soi doivent concourir au développement de l'agentivité personnelle et collective. Les qualités et les attitudes attendues sont l'audace, la persévérance, la confiance en soi, la curiosité, la créativité et la rigueur intellectuelle. Les principes à mettre de l'avant sont les suivants: bien commun, justice, équité, transparence, inclusion, écoresponsabilité, collaboration, engagement, satisfaction personnelle et élévation (plus-être). 


\section{Dimension praxéologique}

La relation d'enseignement doit être horizontale, basée sur la mutualité et favoriser le co-apprentissage. L'éducateur n'est pas un simple facilitateur, il est responsable d'enrichir la compréhension et les représentations des étudiants, de questionner les conceptions erronées, d'insuffler une curiosité intellectuelle, d'encourager l'autonomie et la volonté à s'insérer dans le monde de manière critique. Les enseignants doivent être intéressés à connaitre leurs étudiants et doivent reconnaitre leurs savoirs d'expérience afin de convoquer leurs représentations et conceptions initiales au centre de la situation d'apprentissage. Ils doivent se détacher d'une posture en surplomb qui est celle d'expert détenteur de savoirs neutres et objectifs pour se positionner en tant que co-apprenants intellectuels habités par des valeurs, des principes et des questionnements :

On est des maitres. Pas des égaux. Pour moi l'autorité, ça ne m'intéresse pas. J'essaie toujours de créer une relation avec mes étudiants qui est basée sur le respect. Si tu veux être respecté comme maitre, tu dois respecter ceux à qui tu t'adresses. Ne pas les rabaisser s'ils n'ont pas compris. (Pierre)

En tant que modèles pour leurs étudiants, les enseignants doivent être incarnés et congruents: "Quand il faut enseigner quelque chose d'aussi profond qu'acteur de changement, c'est sûr que, inévitablement, ça prend des gens qui le sont eux-mêmes. » (Simon)

La relation d'apprentissage doit être basée sur une approche réflexive et métacognitive dans laquelle l'objectivation des savoirs s'effectue notamment grâce à l'apport de la subjectivité de l'apprenant, qui est elle-même complexifiée dans la confrontation avec la subjectivité exprimée par ses pairs. D'autre part, l'autonomie de l'apprentissage doit être favorisée par l'utilisation de stratégies d'apprentissage interactives: "Les faire discuter entre eux des questions puissantes, difficiles, émotionnelles. Il faut qu'ils sentent la tension, que même dans un groupe homogène, ils ont des points de vue différents. » (Simon)

7 Une formation d'acteurs de changement doit être intégrée de manière longitudinale et non pas être parachutée de manière ponctuelle et détachée du reste du programme :

Il faut que ces activités d'apprentissage ne soient pas quelque chose de ponctuel, en silo. Il faut que ce soit tout au long du parcours, qu'il y ait une progression des apprentissages, avec des jalons. [...] Inclure à des activités qui existent déjà, enrichir plutôt que de rajouter. (Mireille)

\section{Contribution des étudiants}

Dans cette section nous présentons de manière synthétique les principaux apports issus des entretiens réalisés avec huit étudiantes et étudiants engagés dans divers programmes de formation: génie, gestion, médecine, ergothérapie, environnement, éducation et droit. Encore une fois, ces apports sont présentés selon qu'ils éclairent la dimension formelle, axiologique ou praxéologique de l'éducation envisagée. 


\section{Dimension formelle}

50 Le changement, qui est l'inverse du statu quo, est caractérisé par une dimension scalaire et temporelle. Un acteur de changement est une personne congruente, réflexive et critique animée par une volonté de changer les choses et qui sait influencer et mobiliser des ressources pour attirer l'attention sur une problématique et agir pour sa résolution ou l'amélioration des conditions actuelles. Le changement est orienté vers une amélioration des conditions sociales et environnementales.

51 «Le changement c'est l'inverse du statu quo avec comme postulat de départ, que la situation de départ n'est pas souhaitable ou optimale ou qu'il y a nécessité qu'on ne reste pas dans cette situation-là. Le problème n'est pas nécessairement connu ou reconnu dans la sphère publique. » (Sébastien)

\section{Dimension axiologique}

52 Une formation d'acteur de changement doit mettre l'accent sur le développement de savoir-être (attitudes) et de savoir-faire (habiletés) relatifs au développement du sens de l'agentivité des étudiants. La finalité doit être celle de la transformation des réalités sociétales, mais les buts et les objectifs de la formation ne doivent pas être conditionnés par les résultats de la mise en action. Ce n'est pas l'impact qui doit être mesuré, mais les attitudes et les habiletés. Les attitudes principales à favoriser sont les suivantes: la conscience critique, la perspicacité, la persévérance, la rigueur, la détermination, l'autonomie, le sens de l'initiative et de la responsabilité, l'audace, la congruence et la réflexion éthique (savoir enchâsser ses actions dans un système de valeurs clarifié).

53 En fait, être acteur de changement c'est d'avoir une pensée critique, qui permet de voir les choses autrement, d'avoir la créativité de sortir de ce qui a toujours été fait, d'oser voir les choses différemment. [...] Courage d'oser dire les choses, de faire les choses même si ça va à l'inverse du statu quo. [...] c'est pas juste combien de personnes tu as réussi à atteindre, combien d'écoles tu as réussi à transformer. (Jessica)

54 En ce qui concerne les habiletés, il s'agit principalement de développer des savoirs de stratégie, notamment en sachant comment reconnaitre, prendre en compte et dépasser les rapports de pouvoir pour réussir à influencer les bonnes personnes dans les bonnes circonstances: "Savoir s'entourer, identifier les ennemis pour savoir agir le plus intelligemment possible. » (Sébastien).

\section{Dimension praxéologique}

55 La relation d'enseignement doit être caractérisée par un rapport non hiérarchique dans lequel l'enseignant et les étudiants sont tous co-apprenants au sein d'une communauté d'apprentissage. La relation d'apprentissage doit favoriser une pédagogie active, interactive et expérientielle. Ce sont les réalités sociétales qui doivent être au centre de la situation d'apprentissage. Ainsi, les disciplines et les divers savoirs d'expérience sont mobilisés ensemble afin de construire des savoirs à propos de la réalité qui est posée comme objet de savoirs. Pour favoriser cet apprentissage, l'école doit s'ouvrir sur son milieu et des activités éducatives doivent être vécues hors des murs de l'école. D'autre part, les savoirs ne doivent pas être construits uniquement à travers la dimension cognitive des étudiants, mais doivent également faire appel aux autres dimensions de leur être au monde: affective, sensible, somatique. La relation didactique (relation 
entre la personne enseignante et les objets de savoirs) doit quant à elle mettre davantage l'accent sur une approche critique des savoirs transmis et construits.

Il [l'enseignant] nous posait des questions, mais notre bagage de vie, nos réalités différentes, nos différentes expériences de vie, j'ai vraiment senti que ça l'intéressait. Il était intéressé de savoir ce qu'on avait vécu, c'était quoi les enjeux qu'on avait rencontrés [...] Un de ces profs qui considèrent vraiment ce qu'on a à dire, qui nous prennent dans notre entièreté [...]. Avec lui, je sentais qu'il avait autant à apprendre de nous que nous, de lui. (Sandra)

\section{Discussion : une proposition de repères théoriques et pratiques}

Nous remarquons qu'entre les volets théorique et empirique de cette démarche de recherche, il existe de nombreuses convergences et complémentarités et qu'il n'y a pas à proprement parler de divergences ou d'éléments qui apparaissent irréconciliables.

Notre proposition de repères théoriques et pratiques pour penser et mettre en œuvre des formations d'acteurs de changement en milieu universitaire dans le contexte d'une transition écosociétale s'appuie sur un processus rigoureux d'anasynthèse qui, par un processus interprétatif et dialectique, met en exergue les apports convergents et complémentaires des résultats du volet théorique et de ceux du volet empirique. Nous invitons le lecteur à considérer les repères proposés avec criticité et à les adapter à leurs contextes singuliers d'enseignement-apprentissage.

En ce qui concerne la dimension formelle, nous soutenons qu'un acteur de changement est une personne ayant développé une conscience critique sociale, qui est animée par des valeurs clarifiées et un désir de s'engager individuellement et collectivement pour transformer les réalités sociétales non désirables (enjeux socioécologiques).

Une formation d'acteurs de changement dans le contexte d'une transition écosociétale envisage le changement comme une transformation profonde, radicale et holistique des modes de pensée et d'action des êtres humains. Ce qui est concerné au premier plan c'est l'harmonisation des relations entre les êtres humains et la société, comprise comme une réalité globale qui intègre des réalités socioculturelles et des réalités environnementales, ces réalités devant être comprises comme inextricablement liées.

Pour ce qui est de la dimension axiologique, nous affirmons qu'une formation d'acteurs de changement dans le contexte d'une transition écosociétale doit être résolument tournée et engagée envers la transformation des réalités sociétales qui sont jugées injustes et à la source de dynamiques d'exclusion, d'inégalité, d'aliénation, de domination des humains et des non-humains et des dégradations environnementales. Une telle formation vise constamment à favoriser une compréhension critique du monde, par la recherche des causes structurelles et des schèmes paradigmatiques à l'origine de la reproduction des structures oppressives et aliénantes envers les humains et les non-humains. Dans le même temps, elle s'efforce d'imaginer, de proposer, d'élaborer et de mettre en œuvre des solutions alternatives et ce, dans tous les domaines de la vie en société et à toutes les échelles. Pour y arriver, elle met de l'avant de nombreux principes et valeurs : la justice, la liberté, l'émancipation, l'autonomie, l'écoresponsabilité, l'équité, le respect, l'inclusion, la solidarité, l'engagement, la participation, la transparence, la convivialité, etc. 
62 En ce qui a trait à la dimension praxéologique, mentionnons d'entrée de jeu que l'éducation d'acteurs de changement engagés envers une transition écosociétale en milieu universitaire ne saurait être intégrée de manière parcellaire, en marge du curriculum habituel. Il s'agit plutôt d'une orientation éducationnelle envisagée en tant que dimension transversale de l'ensemble des formations universitaires. Pour mettre en œuvre une telle éducation, nous soutenons que la relation entre l'enseignant et les étudiants doit être basée sur des principes de mutualité et de réciprocité. Le curriculum fait ainsi l'objet d'une négociation entre les étudiants et l'enseignant et doit émerger de la réalité telle que vécue et perçue par les étudiants. Il ne s'agit aucunement de promouvoir une pédagogie libre, dans laquelle nul soutien, direction ou apport d'expérience ne sont apportés par l'enseignant. Il s'agit plutôt de percevoir le rôle de l'enseignant comme celui d'un chercheur interprétatif critique qui se montre intéressé à connaitre et à comprendre les représentations, les présupposés et les conceptions des étudiants relativement aux dimensions du réel étudié. C'est à partir de cette compréhension que l'enseignant peut mieux déterminer quels savoirs académiques et expérientiels il peut apporter afin de les aider à élargir leur compréhension du monde. Dans un tel programme éducatif, les savoirs sont constamment interrogés, mis en contexte et renouvelés. L'enseignant se montre honnête et transparent face aux étudiants en acceptant de partager avec eux sa vision du monde et ses multiples questionnements. Ses propres incertitudes ne sont pas considérées comme une faiblesse, mais plutôt comme une attitude saine et critique à l'égard du monde.

L'enseignant favorise un apprentissage actif et interactif dans lequel les savoirs sont perçus comme des constructions sociales en constant renouvèlement et non pas comme des réalités inexorables. La réalité n'est pas comprise comme étant une fatalité, mais bel et bien comme le résultat des pensées et des actions que les sociétés exercent sur le monde.

Mentionnons enfin que pour éviter une posture autoritaire, tout en ne se déchargeant pas de ses responsabilités d'éducateur, l'enseignant doit savoir doser ses interventions. C'est-à-dire qu'il doit susciter la curiosité intellectuelle des étudiants de manière à faire émerger leurs questionnements critiques. Ainsi, l'enseignant peut apporter des clarifications, corriger des conceptions erronées, enrichir les représentations initiales des étudiants, etc. Il s'agit d'assurer la prise de conscience par les étudiants de leurs représentations, conceptions, présupposés ainsi que de leurs valeurs, puis de favoriser la mise en dialogue de ces éléments au sein du groupe. Les réalités sociétales, au cœur du processus d'apprentissage, sont donc le matériau par lequel les acteurs de la situation éducative peuvent coconstruire du sens et des savoirs.

\section{Conclusion}

65 Afin de partager les réflexions construites au cours de notre recherche réalisée dans le cadre d'une maitrise en environnement, nous avons fait état du contexte entourant la crise socioécologique à laquelle nos sociétés sont confrontées, puis nous avons explicité la pertinence scientifique de notre contribution en relevant les limites et les lacunes quant aux fondements de programmes de formations d'acteurs de changement que nous avons analysés. Cela nous a ensuite permis de formuler une question de recherche pertinente et de clarifier notre objectif principal. Puis, nous avons exposé les principales étapes du processus méthodologique employé. Ensuite, nous avons présenté 
un condensé des apports issus des volets théorique et empirique de la recherche. Enfin, nous avons terminé avec une proposition éducative modélisée pour penser et mettre en œuvre des formations d'acteurs de changement en milieu universitaire dans le contexte d'une transition écosociétale.

En synthèse, former des acteurs de changement exige de prendre un pas de recul afin de redéfinir notre vision de l'éducation en l'envisageant comme une force qui permet de transformer les réalités injustes ainsi que d'imaginer et de coconstruire un monde plus désirable, et ce, grâce à l'éducation de personnes autonomes, conscientes, critiques et engagés dans une praxis sociale qui les transforme en même temps qu'elle transforme le monde.

67 La compréhension critique du monde exige le développement d'une pensée complexe qui aborde de manière holistique les problématiques socioécologiques. Les étudiants et les enseignants doivent s'engager dans une relation mutuelle de création et de recréation du monde. L'enseignant, par sa praxis éducative, est alors amené à affirmer une posture engagée comprenant que tout acte éducatif est politique par essence Les problématiques sociétales sont mises au centre de la situation éducative de manière transdisciplinaire et les co-apprenants s'engagent à les étudier de manière dialogique sous une pluralité de perspectives. Les savoirs d'expérience et la subjectivité sont reconnus et mobilisés. Dès lors, le fossé entre théorie et pratique peut se rétrécir.

Malgré certaines limites inhérentes à la recension des écrits ainsi qu'au nombre et à la diversité des profils de participants, nous croyons que notre analyse permet de dégager de nouvelles significations pour le champ de l'éducation en milieu universitaire dans le contexte contemporain d'une transition écosociétale. Nos propositions pourraient notamment inspirer la conception et la mise à l'essai de projets de formation contextuellement adaptés. Ainsi, des études théoriques et des expérimentations ultérieures pourraient mener à l'élaboration d'un véritable modèle théorique et pratique pouvant orienter un vaste ensemble de formations universitaires d'acteurs de changement engagés envers une transition écosociétale.

\section{BIBLIOGRAPHIE}

AshokaU (s.d). AshokaU. Changemaker campus. Récupéré sur https://ashokau.org/ changemakercampus/

Bélanger, P.R. (2007). L'innovation sociale et ses acteurs : innovations sociales et rapports sociaux. Dans J-L. Klein, (dir.), L'innovation sociale. Émergence et effets sur la transformation de la société (p.413-423). Québec : Presses de l'Université du Québec.

Biesta, G. J.J. (2006). Beyond Learning : Democratic Education for a Human Future. London and New York : Routledge.

Biesta, G. (2017, mai). The Beautiful Risk of Education. Conférence présentée au Schools of tomorrow kick-off conference, Berlin, Allemagne. https://www.youtube.com/watch? $\mathrm{v}=\mathrm{QMqFcVoXnTI}$ 
Bonnici, F. (2020). Becoming a Changemaker : Introduction to Social Innovation. Coursera. Récupéré sur www.coursera.org/learn/social-innovation\#syllabus

Carr, P. R. (2011). Does your Vote Count? Critical Pedagogy and Democracy. New York, NY : Peter Lang Champoux, M. (2021). Former des acteurs de changement à l'université : repères théoriques et pratiques d'une éducation pour le changement dans le contexte d'une transition écosociétale. (Essai de maitrise, Université de Sherbrooke, Sherbrooke, QC) https://savoirs.usherbrooke.ca/handle/11143/18234

Changemaker residency. (2019). Changemaker residency. Changemaker fellows residency. Récupéré sur www.changemakerfellows.com/

Chateauvert, J, Dufort, P., Durand Folco, J., Morales Hudon, A. ; Stambouli, J., Tremblay-Pepin, S. et Wilson, A. (2020). Manuel pour changer le monde. École d'innovation sociale Élisabeth Bruyère. Montréal : LUX éditeur.

Clover, D. E., Jayme, B. de O., Hall, B. L. et Follen, S. (2013). The Nature of Transformation: Environmental Adult Education. SensePublishers.

Delafield, J. et Mukherjee, M. (2016). Organization spotlight. Cultivating changemakers : Social innovation at the heart of education. Childhood Education, 92(2), 155-157.

Dewey, J. (2014). Reconstruction en philosophie (Ser. Folio. essais, 585). Gallimard.

Dewey, J. (2005). La réalité comme expérience. Tracés (9), 83-92.

Echoingreen. (2020). Fellowship. https://echoinggreen.org/fellowship/

Fontan, J-M. (2007). Innovation et changement social. Dans J-L Klein (dir.) L'innovation sociale. Émergence et effets sur la transformation de la société (p.405-412). Québec : Presses de l'Université du Québec.

Fuessel, A.K. (2020). Becoming changemaker institution. A guidebook for how your campus can increase its relevance and resilience and lead in a rapidly changing world. AshokaU [Édition numérique]. Récupéré sur : https://ashokau.org/guidebook/

Freire, P. (1974). Pédagogie des opprimés suivi de Conscientisation et révolution. Paris : François Maspero.

Hackwood college. (2020). Hawkwood Centre for future thinking. Récupéré sur www.hawkwoodcollege.co.uk/about-us/

Habermas, J. (1971). Knowledge and Human Interests. Boston : Beacon Press.

Kelly, D. M. et Brandes, G. M. (2001). Shifting out of « Neutral » : Beginning Teachers' Struggles with Teaching for Social Justice. Canadian Journal of Education / Revue Canadienne de l'éducation, 26(4), 437-454.

Kincheloe, J.L. (2004). Critical Pedagogy. Peter Lang Primer.

Labadie, F. (2005). Modernité et engagement des jeunes. Dans V. Becquet et C. de Linares (dir.), Quand les jeunes s'engagent. Entre expérimentations et constructions identitaires (p. 55-68). Paris : L'Harmattan, collection « Débats Jeunesses ». Récupéré sur https://www.persee.fr/issue/ debaj_1275-2193_2005_ant_16_1

Lefrançois, D., Éthier, M.-A. et Demers, S. (2009). Justice sociale et réforme scolaire au Québec : le cas du programme d'Histoire et éducation à la citoyenneté. Éthique publique, 11(1).

Legendre, R. (2005). Dictionnaire actuel de l'éducation. Montréal : Guérin, 3e édition, 
Maccia, E. (1966). Educational theorizing and curriculum change. Ohio : Educational Theory Center and Social Studies Curriculum Center, Ohio State University. Récupéré sur https:// files.eric.ed.gov/fulltext/ED018308.pdf

Martineau, S. et Simard, D. (2010). Les groupes de discussion. Québec : Presses de l'Université du Québec.

Messier, G. et Dumais, C. (2016). L'anasynthèse comme cadre méthodologique pour la recherche théorique : deux exemples d'application en éducation. Recherches qualitatives, 35(1), 56-75.

Mucchielli, A. (2009). Dictionnaire des méthodes qualitatives en sciences humaines et sociales. Armand Colin.

Nussbaumer, J. et Moulaert, F. (2007). L'innovation sociale au coeur des débats publics et scientifiques. Dans J.L Klein (dir.) L'innovation sociale. Émergence et effets sur la transformation de la société (p.71-88). Québec : Presses de l'Université du Québec.

Pérez-Ibáñez, I. (2018). Dewey's thought on education and social change. Journal of Thought, 52(3 et 4), 19-31.

Sauvé, L. (2011). La dimension politique de l'éducation relative à l'environnement. Un certain vertige. Éducation relative à l'environnement. Regards. Recherches. Réflexions, 9. Récupéré sur https:// journals.openedition.org/ere/1467\#bibliography

Sauvé, L. (1997). Pour une éducation relative à l'environnement : éléments de design pédagogique : guide de développement professionnel à l'intention des éducateurs. Montréal : Guérin. (2 éd.). Récupéré sur http://www.espace-ressources.uqam.ca/images/Documents/Recherche/Monographies/ L.SAUVE(1997).pdf

Sauvé, L. (2017). Une diversité de courants en éducation relative à l'environnement. Dans Barthes, A. et Lange, J.M. (Dir.). Dictionnaire critique des enjeux et concepts des Éducations à. (113-124). Paris : L'Harmattan.

Sauvé, L. et Asselin, H. (2018). Stratégie québécoise d'éducation en matière d'environnement et d'écocitoyenneté. https://www.coalition-education-environnement-ecocitoyennete.org/wpcontent/uploads/2019/07/Strategie-Edition-complete.pdf

Shor, I. (1992). Empowering Education: Critical Teaching for Social Change. Chicago : The University of Chicago Press.

The changemaker project. (2017). The changemaker project. Récupéré sur www.thechangemakerproject.org/

Thésée, G. et Carr, P. R. (2008). L'interculturel en environnement : rencontre de la justice sociale et de la justice environnementale. Éducation canadienne et internationale, 37(1), 45-70.

Thésée, G., Carr, P. R. et Potwora, F. (2016). Le rôle des enseignants dans l'éducation et la démocratie : impacts d'un projet de recherche sur la perception de futurs enseignants. McGill Journal of Education, 50(2-3), 363-387.

Villemagne, C. (2017). Éducation relative à l'environnement des adultes. Observations théoriques et pratiques. Dans L. Sauvé, I. Orellana, C. Villemagne et B. Bader (dir.), Éducation, Environnement, Écocitoyenneté : Repères contemporains (p.157-175). Québec : Presses de l'Université du Québec.

Villemagne, C. (2008). Regard sur l'éducation relative à l'environnement des adultes, Vertigo, la revue électronique en sciences de l'environnement, 8(1). https://journals.openedition.org/ vertigo/1915 
Westheimer, J., et Kahne, J. (2004). What kind of citizen ? The politics of educating for democracy. American educational research journal, 41(2), 237-269.

\section{NOTES}

1. Nous envisageons une transition écosociétale en tant que concept englobant l'ensemble des transformations radicales de nos rapports au monde (en matière de consommation, de production, d'énergie, de transport, de savoirs, de vivre-ensemble) et la transformation de nos valeurs, principes et croyances. Il s'agit d'une transition dont les contours demeurent à définir mais qui s'enracine dans des paradigmes différents de la société actuelle.

2. Dans notre analyse et notre proposition, les éléments associés à la dimension explicative sont apportés de manière transversale aux trois autres dimensions.

3. La chercheure a communiqué directement avec des enseignants et des étudiants connus pour leur engagement envers la formation d'acteurs de changement à l'Université de Sherbrooke. Cet engagement a pu se manifester soit à travers leur participation à des forums, des ateliers ou des conférences sur le sujet dans les mois précédant la recherche.

4. Pour connaitre l'ensemble des résultats et les précisions relatives à la dimension explicative, le lecteur est invité à consulter la version intégrale de la recherche (Champoux, 2021).

5. Pour connaitre les contributions spécifiques de chacun des auteurs (et de certains autres qui ne font pas l'objet de cet article), nous référons le lecteur au texte intégral de l'essai dans lequel une section entière du volet théorique explicite les apports de chacun des auteurs retenus et offre de nombreuses citations directes (Champoux, 2021, p.39-79).

\section{RÉSUMÉS}

Dans le contexte actuel d'une crise socioécologique majeure, former des acteurs de changement apparait pour le moins une orientation éducationnelle accrocheuse. Toutefois, une recherche documentaire a permis de constater que le foisonnement actuel de formations dites d'acteurs de changement (tant en milieu formel que non formel) ne s'appuie pas sur un répertoire d'idées et de théories pertinentes au regard de l'ampleur, de la gravité et de la complexité du contexte de crise plurielle auquel nos sociétés se trouvent confrontées. Cet article a pour objectif de présenter un condensé des résultats d'une recherche de maitrise en environnement qui s'est penchée sur l'élaboration de repères théoriques et pratiques pour penser et mettre en œuvre des formations d'acteurs de changement en milieu universitaire dans le contexte d'une transition écosociétale. Les résultats mettent en évidence les dimensions subversive, critique et émancipatrice de telles formations.

In the context of a major socio-ecological crisis, such as the one facing our current societies, educating agents of change appears at least to be an eye-catching educational orientation. However, a documentary research has shown that the current proliferation of training courses known as agents of change or changemakers (both in formal and non-formal settings) is not based on a repertoire of ideas and theories relevant to the scope, gravity and complexity of the context of plural crisis which our societies are confronted with. The objective of this article is to present a summary of the results of a master's degree research in environmental studies that 
looked at the development of theoretical and practical benchmarks for thinking and implementing programs seeking to educate change agents in academia, in the context of an ecosocietal transition. The results highlight the subversive, critical and emancipatory dimensions of such training.

\section{INDEX}

Mots-clés : pédagogie critique, éducation supérieure, crise socioécologique, éducation relative à l'environnement, éducation à la démocratie et à la citoyenneté, innovation sociale

Keywords : critical pedagogy, higher education, socioecological crisis, environmental education, democracy and citizenship education, social innovation

\section{AUTEUR}

\section{MÉLANIE CHAMPOUX}

Mélanie Champoux est titulaire d'une maitrise en environnement et d'un diplôme en éducation relative à l'environnement. Elle poursuit actuellement ses études à travers le programme en éducation transformatoire à la démocratie et à la citoyenneté mondiale à l'Université du Québec en Outaouais et elle amorce un parcours doctoral en éducation à l'Université de Sherbrooke. Ses intérêts de recherche se portent sur les orientations éducationnelles des programmes de formations universitaires dans le contexte d'une transition écosociocritique. 